\title{
miR-27b attenuates apoptosis induced by transmissible gastroenteritis virus (TGEV) infection via targeting runt- related transcription factor 1 (RUNX1)
}

Xiaomin Zhao, Xiangjun Song, Xiaoyuan Bai, Naijiao Fei, Yong Huang, Zhimin Zhao, Qian Du, Hongling Zhang, Liang Zhang, Dewen Tong

Transmissible gastroenteritis virus (TGEV), belonging to the coronaviridae family, is the key cause of the fatal diarrhea of piglets and results in many pathological processes. microRNAs (miRNAs) play a key role in the regulation of virus-induced apoptosis. During the process of apoptosis induced by TGEV infection in PK-15 cells, the miR-27b is notably down-regulated. Thus, we speculate that miR-27b is involved in regulating the process of apoptosis in PK-15 cells. In this study we demonstrated that the over-expression of miR$27 \mathrm{~b}$ led to the inhibition of TGEV-induced apoptosis, reduction of Bax protein level, and decrease of caspase- 3 and -9 activities. Conversely, silencing of miR-27b by miR-27b inhibitors enhanced apoptosis via up-regulating Bax expression and promoting the activities of caspase-3 and -9 in TGEV-infected cells. Subsequently, the runt-related transcription factor 1 (RUNX1) is a candidate target of miR-27b predicted by bioinformatics search. We further identified that the miR-27b directly bound to the 3' UTR of RUNX1 mRNA and suppressed RUNX1 expression, which indicates RUNX1 is the direct target gene of miR-27b. The over-expression of RUNX1 increased apoptosis and knockdown RUNX1blocked apoptosis of viral-infected cells via regulating Bax expression and the activities of caspase- 3 and -9 . Our data reveal that miR-27b may repress the mitochondrial pathway of apoptosis by targeting RUNX1, indicating that TGEV may induce apoptosis via down-regulating miR-27b and that miR-27b may act as a target for therapeutic intervention. 
3 Xiaomin Zhao a, \#, Xiangjun Song a, \#, Xiaoyuan Bai a, Naijiao Fei a, Yong Huang a, Zhimin Zhao a, Qian Du a,

4 Hongling Zhang a, Liang Zhang a , Dewen Tong a,*

5

6

${ }^{a}$ College of Veterinary Medicine, Northwest A\&F University, Yangling, Shaanxi 712100, P.R. China

7

E-mails: xiaominz2012@163.com (XZ); songxiangjun1007@126.com (XS); bxybxy1014@163.com (XB);

396726686@qq.com (NF); huangyong@nwsuaf.edu.cn (YH); 13032920832@163.com (ZZ); dewey3600@163.com

(QD); ready866281@163.com (HZ); zhanglianghappyus@163.com (LZ); dwtong@nwsuaf.edu.cn (DT).

* Author to whom correspondence should be addressed; E-Mail: dwtong@nwsuaf.edu.cn (DT); Tel: +86-29-

\# These authors contributed equally to this work. 


\section{Abstract}

23 Transmissible gastroenteritis virus (TGEV), belonging to the coronaviridae family, is the key cause of the fatal diarrhea of piglets and results in many pathological processes. microRNAs (miRNAs) play a key role in the regulation of virus-induced apoptosis. During the process of apoptosis induced by TGEV infection in PK-15 cells, the miR-27b is notably down-regulated. Thus, we speculate that miR-27b is involved in regulating the process of apoptosis in PK-15 cells. In this study we demonstrated that the over-expression of miR-27b led to the inhibition of TGEV-induced apoptosis, reduction of Bax protein level, and decrease of caspase-3 and -9 activities. Conversely, silencing of miR-27b by miR-27b inhibitors enhanced apoptosis via up-regulating Bax expression and promoting the activities of caspase-3 and -9 in TGEV-infected cells. Subsequently, the runt-related transcription factor 1 (RUNX1) is a candidate target of miR-27b predicted by bioinformatics search. We further identified that the miR-27b directly bound to the 3' UTR of RUNX1 mRNA and suppressed RUNX1 expression, which indicates RUNX1 is the direct target gene of miR-27b. The over-expression of RUNX1 increased apoptosis and knockdown RUNX1 blocked apoptosis of viral-infected cells via regulating Bax expression and the activities of caspase-3 and -9. Our data reveal that miR-27b may repress the mitochondrial pathway of apoptosis by targeting RUNX1, indicating that TGEV may induce apoptosis via down-regulating miR-27b and that miR-27b may act as a target for therapeutic intervention.

Key words: miR-27b; transmissible gastroenteritis virus; apoptosis; RUNX1

Introduction

TGEV, a member of Coronaviridae family, is an enveloped virus with a positive-sense single-stranded RNA 
43

genome (Weiss \& Leibowitz 2011). TGEV infection primarily causes transmissible gastroenteritis (TGE) that is characterized by highly contagious and fatal gastroenteritis for pigs of all ages, especially for piglets under 2 weeks old (Chae et al. 2000; Kim \& Chae 2001).

Apoptosis is a process of self-destruction in response to a variety of stimuli such as viral infection. Infection of coronavirus such as porcine epidemic diarrhea virus (PEDV) (Kim \& Lee 2014), infectious bronchitis virus (IBV) (Li et al. 2007), severe acute respiratory syndrome coronavirus (SARS-CoV) (Krahling et al. 2009), may result in host cell apoptosis. We have reported that TGEV infection induced apoptosis via mitochondria mediated apoptotic pathway in PK-15 cells (Ding et al. 2012; Ding et al. 2013).

miRNAs are a class of small non-coding RNA molecules and may regulate gene expression at post-transcription level (Bartel 2009). Numerous experimental studies have demonstrated that miRNAs play important roles in cell apoptosis (Wilson \& Doudna 2013). miR-29b effectively inhibits apoptosis via directly targeting caspase-7 and nuclear apoptosis inducing factor 1 (NAIF1) in Madin-Darby Bovine Kidney (MDBK) cells infected with bovine viral diarrhea virus (BVDV) (Fu et al. 2014). miR-27b promotes doxorubicin-induced apoptosis in hepatoblastoma cell line (HepG2) (Mu et al. 2015). Moreover, miR-27b is an endogenous inhibitory factor of apoptotic peptidase activating factor 1 (Apaf-1) expression and decreases the apoptotic rate of neurons(Chen et al. 2014). miRNAs are also involved in regulating the process of virus-induced apoptosis (Smith et al. 2012; Zhang et al. 2013). Hs_154 promotes WNV-mediated apoptosis via inhibiting the expression of CCCTC-binding factor (CTCF) and the epidermal growth factor receptor (EGFR) (Smith et al. 2012). Our previous studies showed that miR-27b was significantly down-regulated during TGEV-induced apoptosis (Song et al. 2015). Thus, we supposed that miR$27 \mathrm{~b}$ may play a role in apoptosis induced by TGEV infection. In the present study, we investigated the role of miR-27b in TGEV-induced apoptosis in PK-15 cells and demonstrated that the miR-27b attenuated TGEV-induced 
64 apoptosis by targeting RUNX1, suggesting TGEV may use miR-27b to regulate apoptosis in PK-15 cells.

65

\section{Methods}

Antibodies, Cells and virus. Monoclonal RUNX1 (MAB23991) was purchased from R\&D Systems (R\&D Systems, MN, US). Monoclonal antibodies against Bax (sc-23959) and $\beta$-actin (sc-69879) were purchased from Santa Cruz Biotechnology (Santa Cruz, Inc., CA, US). Horseradish peroxidase (HRP)-conjugated secondary antibody was purchased from Pierce (Pierce, Rockford, IL, US). PK-15 cells were obtained from ATCC (CCL-33) and cultured in Dulbecco's Minimal Essential Medium (DMEM) supplemented with 10\% fetal bovine serum (Gibco BRL, Gaithersburg, MD, US), $100 \mathrm{IU}$ of penicillin, and $100 \mathrm{mg}$ of streptomycin per $\mathrm{ml}$, at $37^{\circ} \mathrm{C}$ in a $5 \% \mathrm{CO}_{2}$ atmosphere incubator. The TGEV Shaanxi strain was isolated from TGEV-infected piglets by Ding L et al (Ding 2011).

miRNAs quantification by real-time PCR. Total RNA was extracted using Trizol reagent (Invitrogen, Carlsbad, CA, US) from PK-15 cells Reverse transcription reactions and real-time PCR were performed as described previously(Song et al. 2015). The relative quantification of miRNAs was normalized to U6 using the two-ddCt method (Livak \& Schmittgen 2001).

Flow cytometry analysis. Annexin V-FITC Apoptosis Kit (Invitrogen, Carlsbad, CA, US) was used to detect apoptosis according to the manufacturer's protocol. Briefly, cells were washed twice with ice-cold PBS and resuspended in $500 \mu \mathrm{L} 1 \times$ Annexin $\mathrm{V}$ binding buffer followed by adding $5 \mu \mathrm{L}$ Annexin V-FITC and $5 \mu \mathrm{L}$ PI. After incubated for $30 \mathrm{~min}$ at room temperature, analysis was done by flow cytometry (Beckman Coulter, CA. US).

Caspase activity assay. Caspases activities were measured by colorimetric assay kits (Keygen Biotech, Nanjing, China) following the manufacture's protocol. Briefly, protein concentrations were measured by BCA Protein Assay Reagent (Vazyme Biotech Nanjing, China). Then $200 \mathrm{mg}$ protein of each sample was incubated with each caspase substrate at $37{ }^{\circ} \mathrm{C}$ in a microplate for $4 \mathrm{~h}$. Absorbance at the wavelength of $405 \mathrm{~nm}$ was read in microplate 
spectrophotometer (Infinite 200 PRO NanoQuant, Tecan, Switzerland).

Luciferase reporter experiments. The 3' UTR sequence of RUNX1 mRNA containing the target sites of miR-27b was amplified by PCR using the primers of supplementary Table S1. The 3' UTR of RUNX1 mRNA was cloned into psiCHECK-2 (Promega, Madison, WI, US) between Xho I and Not I cloning sites to obtain the wild type plasmid RUNX1-WT. The binding sequences of miR-27b seed region in 3' UTR of RUNX1-WT were mutated following a mutagenesis protocol (Heckman \& Pease 2007) to generate RUNX1-mut. miR-27b mimics, miRNA mimics control, miR-27b inhibitors and miRNA inhibitors control were synthesized by Ribo Biotech (RiboBio, Guangzhou, China). miR-27b inhibitors were modified with 2'-O-methyl. The sequences of miRNA in this study were shown in supplementary Table S2. For the luciferase reporter assay, PK-15 cells were grown in 24-well plates and then co-transfected with plasmid RUNX1-WT (or RUNX1-mut) and miR-27b mimics (or miR-27b inhibitors) using Lipofectamine 3000 (Invitrogen, Carlsbad, CA, US). The luciferase activities were detected at $48 \mathrm{~h}$ post transfection (hpt) using a Dual-Glo ${ }^{\circledR}$ Luciferase Assay System (Promega, Madison, WI, USA) following the manufacturer's manual.

RNA interference. Three siRNAs (siRUNX1-1, siRUNX1-2, siRUNX1-3) of RUNX1 were synthesized by GenePharma (GenePharma, Shanghai, China). The most effective siRNA (si-RUNX1-2) was applied for the further experiments. PK-15 cells were transfected with 100 nM RUNX1-specific siRUNX1-2 (Supplementary Table S2) or irrelevant siRNA using Lipofectamine 3000 (Invitrogen, Carlsbad, CA, US).

Cell viability assay. Cell viability was detected using Cell Counting Kit-8 (CCK-8) (Vazyme Biotech Nanjing, China). Briefly, cells were seeded in 96 -well culture plate and cultured at $37{ }^{\circ} \mathrm{C}$ in a humidified atmosphere with $5 \%$ $\mathrm{CO}_{2} .12 \mathrm{~h}$ later, cells were transfected with $100 \mathrm{nM}$ siRUNX1-2 or irrelevant siRNAs. The cell viability was tested following the manufacturer's manual at $48 \mathrm{hpt}$. 
106 Vector construction. To construct the RUNX1 expression plasmid, the full-length RUNX1 gene was amplified by

PCR and was cloned into plasmid pCI-neo (Promega, Madison, WI, US) between EcoR I and Not I cloning sites to gain pCI-neo-RUNX1. The primer sequences of PCR are shown in supplementary Table S1. PK-15 cells were transfected with pCI-neo (control) or pCI-neo-RUNX1. Cells were infected with TGEV at multiplicity of infection (MOI) of 1 at $48 \mathrm{~h}$ post infection (hpi) .

111 Quantification of mRNAs by real-time PCR. The total RNA was extracted using Trizol reagent (Invitrogen,

112 Carlsbad, CA, US) according to manufacturer's instructions. A total of $2 \mu \mathrm{g}$ of RNA was transcribed into cDNA using the First-strand cDNA synthesis kit (Invitrogen, Carlsbad, CA, US). Real-time PCR was carried out using the AccuPower® $2 \times$ Greenstar qPCR Master mix (Bioneer, Daejeon, Korea) on Bio-Rad iQ5 Real-Time PCR System.

The relative fold changes were calculated using the 2-ddCt method. The real-time PCR primers are shown in supplementary Table S3.

Western blot analysis. Cells were treated with Radioimmunoprecipitation Assay (RIPA) lysis buffer containing phenylmethyl sulfonylfluoride (PMSF). Protein concentration was tested with BCA Protein Assay Reagent (Pierce, and subsequently transferred to a polyvinylidene difluoride (PVDF) membrane (Millipore Corp, Atlanta, GA, US).

The membrane was blocked with 5\% non-fat dry milk for $2 \mathrm{~h}$ at room temperature and then incubated with the primary antibodies overnight at $4{ }^{\circ} \mathrm{C}$. The HRP-conjugated secondary antibodies were used to incubate for $1 \mathrm{~h}$ at room temperature. The blotting was visualized using Enhanced Chemiluminescence (ECL) reagent.

Statistics. All data are representative of results from at least 3 separate experiments. All data points are the average of triplicates, with error bars representing standard deviations. The difference between two groups was statistically 
127

128

129

130

131

132

133

134

135

136

137

138

140

141

142

143

144

145

146

147

\section{Results}

\section{miR-27b attenuates TGEV-induced apoptosis}

To investigate whether miR-27b is involved in the apoptosis induced by TGEV, the cells were transfected with miR-

$27 \mathrm{~b}$ mimics or miR-27b inhibitors and infected with TGEV at a MOI of 1 at $24 \mathrm{hpt}$. The apoptotic rate was analyzed

by flow cytometry at 24 and 48 hpi. The results showed that the over-expression of miR-27b led to a decrease of apoptotic rate and the down-expression of miR-27b increased apoptotic rate at 24 and 48 hpi (Fig. 1A), indicating that miR-27b attenuated apoptosis induced by TGEV infection in PK-15 cells. We previously found that TGEV infection could cause PK-15 cell apoptosis through mitochondria-mediated pathway and FasL-mediated apoptotic pathway (Ding et al. 2012). Therefore, we analyzed caspase-9 activity of mitochondria-mediated pathway and caspase-8 activity of FasL-mediated apoptotic pathway. However, miR-27b did not affect the caspase-8 activity during TGEV infection (data not shown). As expected, the caspase-9 activity was decreased by miR-27b mimics and up-regulated by miR-27b inhibitors in the TGEV-infected PK-15 cells (Fig. 1B). Moreover, we investigated the effect of miR-27b on caspases-3 activity in TGEV-infected PK-15 cells. The caspase-3 activity was decreased by miR-27b mimics and increased by miR-27b inhibitors in the TGEV-infected cells compared with control (Fig. 1C).

Taken together, these results suggest that miR-27b plays a negative role in the TGEV-induced apoptosis in PK-15 cells via mitochondria-mediated pathway.

\section{RUNX1 is the direct target of miR-27b}

To identify the targets that affect TGEV-induced apoptosis, we predicted the targets of miR-27b using TargetScan and miRanda and found that RUNX1 was a potential target of miR-27b (Song et al. 2015). Moreover, RUNX1 was reported to regulate apoptosis (Wu et al. 2012). Thus, we investigated the direct interaction between miR-27b and $3^{\prime}$ UTR of RUNX1 mRNA through dual-luciferase assay. The sequence of the 3' UTR of RUNX1 was obtained by 
148 PCR and the two binding sites of miR-27b seed region in the 3' UTR of RUNX1 were mutated with a 3-bp

149 substitution. The 3' UTR of RUNX1 and its mutational version were respectively sub-cloned into the 3' UTR of the

150 Renilla luciferase dual-luciferase plasmid psiCHECK-2 (Fig. 2A and B). The constructs were co-transfected into

151 PK-15 cells with miR-27b mimics (or negative control) or miR-27b inhibitors (or negative control). Compared with

the miRNA mimics control, introduction of miR-27b mimics decreased the RUNX1-WT reporter activity (Fig. 2C).

To determine whether miR-27b inhibits RUNX1 expression, miR-27b mimics or miRNA mimics control were

transfected into PK-15 cells. RUNX1 expression was assessed by western blot. The result showed that miR-27b

decreased expression of RUNX1 in PK-15 cells compared with the control (Fig. 2D). Together, our data

conclusively demonstrate that RUNX1 is a direct target of miR-27b in PK-15 cells.

miR-27b attenuates apoptosis though regulating mitochondrial pathway

RUNX1 shows transactivate effect on Bax expression (Wu et al. 2012). We previously found that TGEV infection

could cause PK-15 cell apoptosis through mitochondria-mediated pathway (Ding et al. 2012). Therefore, we

proposed a hypothesis that miR-27b could restrain TGEV-induced apoptosis of PK-15 through mitochondria-

mediated pathway by decreasing expression of Bax. In order to confirm this hypothesis, we analyzed the effect of miR-27b on Bax. At 24 hpt of miR-27b mimics or miR-27b inhibitors, the cells were infected with TGEV at 1 MOI.

The mRNA and protein levels of Bax were analyzed by real-time PCR and western blotting at 24 and 48 hpi. Over-

$27 \mathrm{~b}$ attenuates TGEV-induced apoptosis by suppressing Bax.

RUNX1 enhances TGEV-induced apoptosis

To investigate the effect of RUNX1 on apoptosis, we silenced and over-expressed RUNX1 respectively using 
siRUNX1 and pCI-neo-RUNX1. The RUNX1 mRNA and protein levels were remarkably reduced by siRUNX1-2 (Fig. 4A and B), however the cell viability was not affected in comparison with irrelevant siRNA (Fig. 4C), indicating that the RUNX1 gene was knocked down in PK-15 cells by the siRUNX1-2. In addition, RUNX1 was notably over-expressed using pCI-neo-RUNX1 (Fig. 4D). To determine the effect of RUNX1 on Bax, the RUNX1 were silenced with siRUNX1-2 and over-expressed by pCI-neo-RUNX1 followed by infection with TGEV. The Bax expression was decreased by knockdown of RUNX1 and up-regulated by over-expression of RUNX1 (Fig. 4E). The activities of caspase- 9 and -3 were respectively reduced and increased by silence and over-expression of RUNX1 (Fig. 4F and 4G). Therefore, RUNX1 promoted TGEV-induced apoptosis of PK-15 cells. Taken together, our findings suggest that miR-27b shows a negative regulatory effect on TGEV-induced apoptosis of PK-15 cells via targeting RUNX1.

\section{Discussion}

Accumulating evidence has shown that miRNAs play an important role in apoptosis induced by virus infection (Fu et al. 2014; Guan et al. 2012; Smith et al. 2012). We previously found that TGEV infection induced apoptosis in PK15 cells via mitochondria-mediated apoptosis pathway (Ding et al. 2012). In addition, we reported that miR-27b was significantly down-regulated during apoptosis triggered by TGEV infection in PK-15 cells (Song et al. 2015). However, the role of miR-27b in the process of apoptosis induced by TGEV infection is unclear. Here we proved that miR-27b suppressed the TGEV-induced apoptosis via regulating mitochondrial pathway.

During the viral infection, miRNAs may regulate the interaction between virus and host cell via targeting viral or cellular genes. miR-181c, which is down-regulated by HCV infection in hepatocytes, suppresses homeobox A1 expression (HOXA1) and its downstream molecules STAT3 and STAT5 to regulate cell growth by targeting E1 and NS5A sequences of HCV (Mukherjee et al. 2014). miR-1236 represses HIV infection by inhibiting transcription 
190

192

of HIV-1 viral protein R-binding protein (VprBP ) in monocytes (Ma et al. 2014). We assessed the effects of miR$27 \mathrm{~b}$ on the replication and transcription of TGEV genes and found that the replication and transcription of TGEV structural and non-structural genes were not affected by miR-27b (Data are not shown). We tested the effects of miR-27b on apoptotic rate of TGEV-infected PK-15 cells. The results showed that miR-27b decreased TGEVinduced apoptosis. We previously reported that miR-27b was decreased by TGEV infection (Song et al. 2015), so we propose that miR-27b may present an antagonistic effect with TGEV infection on apoptosis via regulating mitochondrial pathway. An analogous situation has been reported that miR-27b alleviate hypoxia-induced neuronal apoptosis(Chen et al. 2014). It indicates that miR-27b not only down-regulates cancer cell apoptosis, it also suppresses virus-induced apoptosis.

Apoptosis is primarily modulated by two pathways, extrinsic pathway and intrinsic pathway (mitochondrionmediated), which regulate cell death involving the continuous activation of caspases (Lavrik 2010). Caspase-8 and caspase-9 are factors of extrinsic pathway and mitochondrial pathway respectively (Sakamaki et al. 2015; Thornberry \& Lazebnik 1998). Bax, a member of the BCL-2 family, functions as an apoptotic activator to activate caspase-9 and -3 (Chipuk et al. 2010; Ding et al. 2012). In this study, we showed that miR-27b inhibited Bax expression and reduced the activities of caspase- 9 and -3 during TGEV-induced apoptosis. We demonstrated that miR-27b regulated apoptosis via down-regulation the expression of Bax and activation of caspase- 9 and caspase-3, implying that miR-27b are involved in regulating TGEV-induced apoptosis via regulating mitochondrial pathway. The miRNAs suppress gene expression by imperfectly binding to the $3^{\prime}$ UTR of target mRNA in mammalian cells. The imprecise binding make each miRNA has the ability to target multiple mRNAs. For example, miR-27b could suppress adipogenesis in hMADS cells by targeting peroxisome proliferator-activated receptor gamma (PPAR $\gamma$ ) (Karbiener et al. 2009) and promote differentiation of myeloblasts through targeting RUNX1 (Feng et al. 2009). In 
the present study, we confirmed that RUNX1 is the target of miR-27b during TGEV infection, while whether miR-

$27 \mathrm{~b}$ regulates apoptosis via RUNX1 is unclear. RUNX1 is a transcription factor, and play important roles in

regulating the growth, development, and/or differentiation of multiple lineages of haematopoietic cells (Feng et al.

2009). We found over-expression of RUNX1 reduced Bax and decreased the activities of caspase-9 and -3 , and

silencing RUNX1 up-regulated Bax and increased the activities of caspase-9 and -3 . This suggests that miR-27b

regulates TGEV-induced apoptosis via targeting RUNX1, which is an important supplement of RUNX1 function.

In summary, our finding suggests miR-27b represses TGEV-induced apoptosis by directly targeting RUNX1, which

may have potential for therapeutic strategies.

\section{References}

Bartel DP. 2009. MicroRNAs: Target Recognition and Regulatory Functions. Cell 136:215-233.

Chae C, Kim O, Choi C, Min K, Cho WS, Kim J, and Tai JH. 2000. Prevalence of porcine epidemic diarrhoea virus and transmissible gastroenteritis virus infection in Korean pigs. Veterinary Record 147:606-608.

Chen Q, Xu J, Li L, Li H, Mao S, Zhang F, Zen K, Zhang CY, and Zhang Q. 2014. MicroRNA-23a/b and microRNA-27a/b suppress Apaf-1 protein and alleviate hypoxia-induced neuronal apoptosis. Cell Death and Disease 5:e1132.

Chipuk JE, Moldoveanu T, Llambi F, Parsons MJ, and Green DR. 2010. The BCL-2 family reunion. Molecular cell 37:299-310.

Ding L, Chen, G.D., Xu, X.G., Tong, D.W. 2011. Isolation and identification of porcine transmissible gastroenteritis virus Shaanxi strain and sequence analysis of its N gene. Chin J Vet Med 47:09-11.

Ding L, Xu X, Huang Y, Li Z, Zhang K, Chen G, Yu G, Wang Z, Li W, and Tong D. 2012. Transmissible gastroenteritis virus infection induces apoptosis through FasL- and mitochondria-mediated pathways. Veterinary Microbiology 158:12-22.

Ding L, Zhao X, Huang Y, Du Q, Dong F, Zhang H, Song X, Zhang W, and Tong D. 2013. Regulation of ROS in transmissible gastroenteritis virus-activated apoptotic signaling. Biochemical and Biophysical Research Communications 442:33-37.

Feng J, Iwama A, Satake M, and Kohu K. 2009. MicroRNA-27 enhances differentiation of myeloblasts into granulocytes by post-transcriptionally downregulating Runx1. Br J Haematol 145:412-423.

Fu Q, Shi H, Shi M, Meng L, Zhang H, Ren Y, Guo F, Jia B, Wang P, Ni W, and Chen C. 2014. bta-miR-29b attenuates apoptosis by directly targeting caspase-7 and NAIF1 and suppresses bovine viral diarrhea virus replication in MDBK cells. Canadian Journal of Microbiology 60:455-460. 
Guan Z, Shi N, Song Y, Zhang X, Zhang M, and Duan M. 2012. Induction of the cellular microRNA-29c by influenza virus contributes to virus-mediated apoptosis through repression of antiapoptotic factors BCL2L2. Biochemical and Biophysical Research Communications 425:662-667.

Heckman KL, and Pease LR. 2007. Gene splicing and mutagenesis by PCR-driven overlap extension. Nature Protocols 2:924-932.

Karbiener M, Fischer C, Nowitsch S, Opriessnig P, Papak C, Ailhaud G, Dani C, Amri EZ, and Scheideler M. 2009. microRNA miR-27b impairs human adipocyte differentiation and targets PPAR gamma. Biochem Biophys Res Commun 390:247-251.

Kim B, and Chae C. 2001. In situ hybridization for the detection of transmissible gastroenteritis virus in pigs and comparison with other methods. Can J Vet Res 65:33-37.

Kim Y, and Lee C. 2014. Porcine epidemic diarrhea virus induces caspase-independent apoptosis through activation of mitochondrial apoptosis-inducing factor. Virology 460-461:180-193.

Krahling V, Stein DA, Spiegel M, Weber F, and Muhlberger E. 2009. Severe Acute Respiratory Syndrome Coronavirus Triggers Apoptosis via Protein Kinase R but Is Resistant to Its Antiviral Activity. J Virol 83:2298-2309.

Lavrik IN. 2010. Systems biology of apoptosis signaling networks. Current Opinion in Biotechnology 21:551-555.

Li FQ, Tam JP, and Liu DX. 2007. Cell cycle arrest and apoptosis induced by the coronavirus infectious bronchitis virus in the absence of p53. Virology 365:435-445.

Livak KJ, and Schmittgen TD. 2001. Analysis of Relative Gene Expression Data Using Real-Time Quantitative PCR and the 2- $\Delta \Delta \mathrm{CT}$ Method. Methods 25:402-408.

Ma L, Shen CJ, Cohen EA, Xiong SD, and Wang JH. 2014. miRNA-1236 inhibits HIV-1 infection of monocytes by repressing translation of cellular factor VprBP. PLoS One 9:e99535.

Mu W, Hu C, Zhang H, Qu Z, Cen J, Qiu Z, Li C, Ren H, Li Y, He X, Shi X, and Hui L. 2015. miR-27b synergizes with anticancer drugs via p53 activation and CYP1B1 suppression. Cell Research 25:477-495.

Mukherjee A, Shrivastava S, Bhanja Chowdhury J, Ray R, and Ray RB. 2014. Transcriptional suppression of miR181 c by hepatitis $\mathrm{C}$ virus enhances homeobox A1 expression. $J$ Virol 88:7929-7940.

Osthus RC, Karim B, Prescott JE, Smith BD, McDevitt M, Huso DL, and Dang CV. 2005. The Myc target gene JPO1/CDCA7 is frequently overexpressed in human tumors and has limited transforming activity in vivo. Cancer Res 65:5620-5627.

Sakamaki K, Imai K, Tomii K, and Miller DJ. 2015. Evolutionary analyses of caspase-8 and its paralogs: Deep origins of the apoptotic signaling pathways. Bioessays 37:767-776.

Smith JL, Grey FE, Uhrlaub JL, Nikolich-Zugich J, and Hirsch AJ. 2012. Induction of the Cellular MicroRNA, Hs_154, by West Nile Virus Contributes to Virus-Mediated Apoptosis through Repression of Antiapoptotic Factors. Journal of virology 86:5278-5287.

Song X, Zhao X, Huang Y, Xiang H, Zhang W, and Tong D. 2015. Transmissible Gastroenteritis Virus (TGEV) Infection Alters the Expression of Cellular MicroRNA Species That Affect Transcription of TGEV Gene 7. International Journal of Biological Sciences 11:913-922.

Thornberry NA, and Lazebnik Y. 1998. Caspases: Enemies within. Science 281:1312-1316.

Weiss SR, and Leibowitz JL. 2011. Coronavirus Pathogenesis. 81:85-164.

Wilson RC, and Doudna JA. 2013. Molecular mechanisms of RNA interference. Annu Rev Biophys 42:217-239.

Wu D, Ozaki T, Yoshihara Y, Kubo N, and Nakagawara A. 2012. Runt-related Transcription Factor 1 (RUNX1) 
283

284

285

286
Stimulates Tumor Suppressor p53 Protein in Response to DNA Damage through Complex Formation and Acetylation. Journal of Biological Chemistry 288:1353-1364.

Zhang Y, Chen N, Zhang J, and Tong Y. 2013. Hsa-let-7g miRNA targets caspase-3 and inhibits the apoptosis induced by ox-LDL in endothelial cells. International journal of molecular sciences 14:22708-22720. 


\section{Figure 1 (on next page)}

Fig. 1. The effect of miR-27b on apoptosis induced by TGEV.

(A) Effect of the miR-27b mimics and inhibitors on apoptosis induced by TGEV. The apoptosis of PK-15 was analyzed via flow cytometry at 24 and 48 hpi. (B) Detection of caspase-9 activities in PK-15 cells at 24 and 48 hpi. (C) Detection of caspase-3 activities in cells at 24 and 48 hpi.* $\mathrm{P}<0.05$ in comparison with the control. ${ }^{* *} \mathrm{P}<0.01$ in comparison with the control. 
A
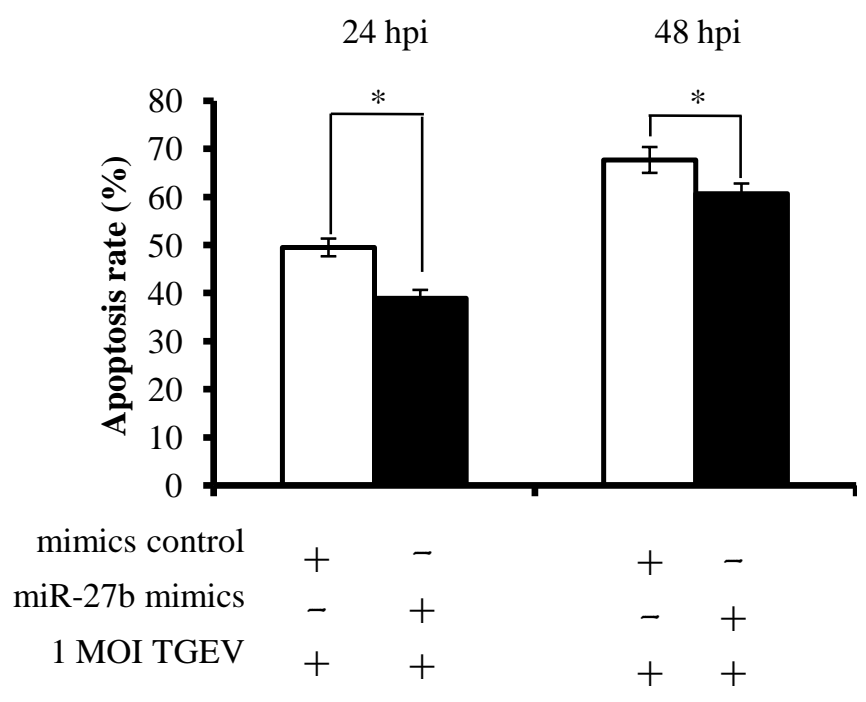

B

24 hpi

48 hpi

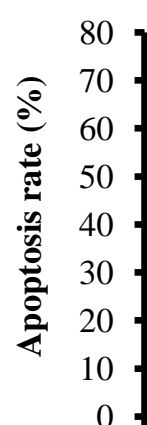

24 hpi

48 hpi
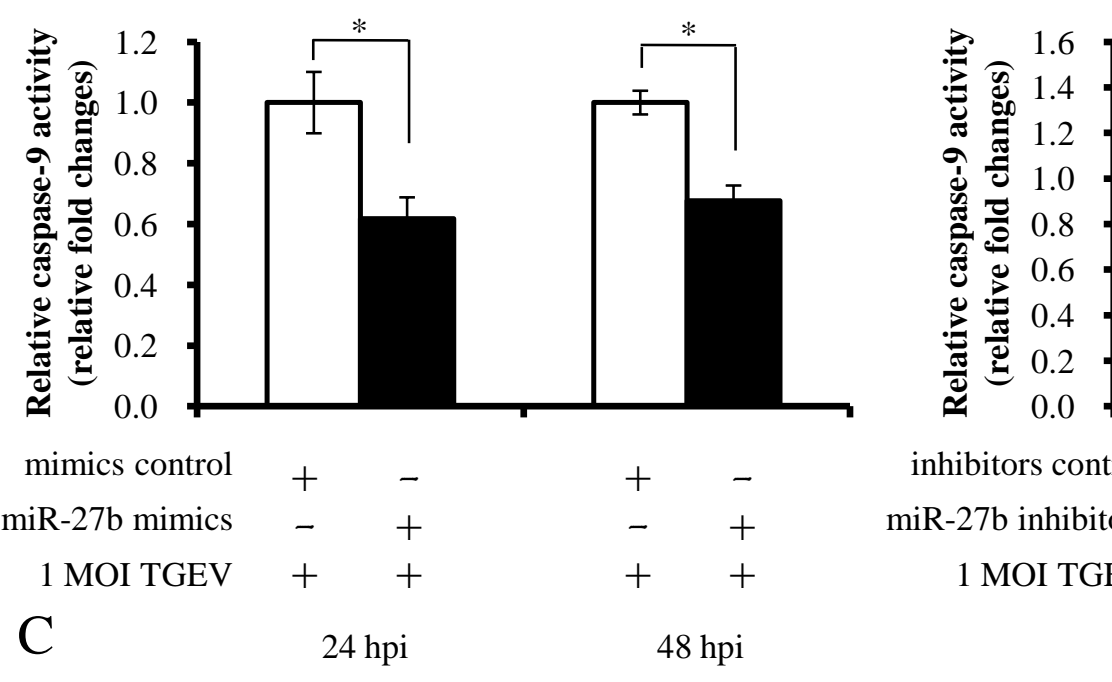

24 hpi

48 hpi
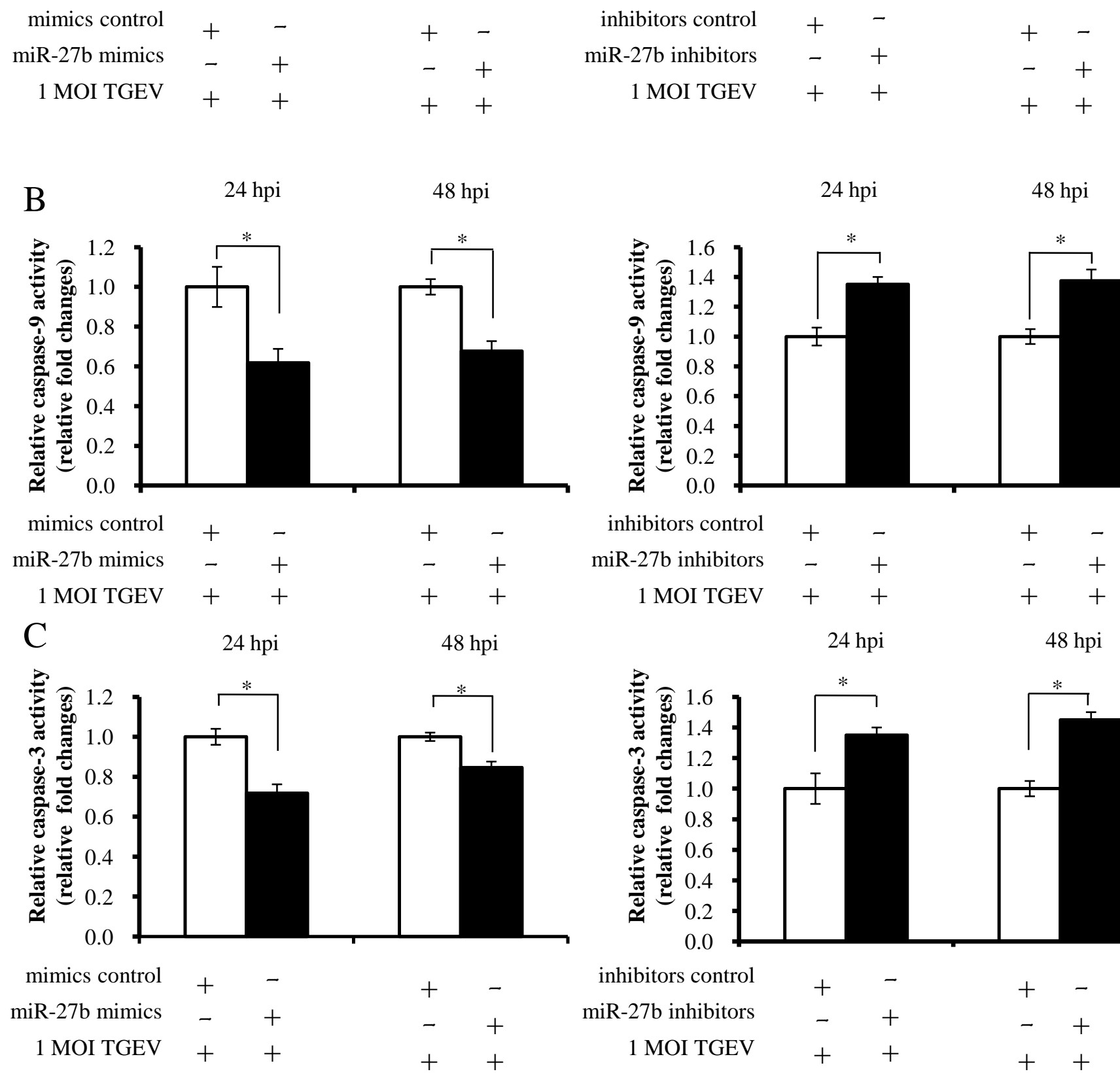


\section{Figure 2 (on next page)}

Fig. 2. miR-27b directly targets the 3' UTR of RUNX1 mRNA.

(A) Bioinformatic prediction of interaction between miR-27b and the 3' UTRs of swine RUNX1. For each schematic, the upper sequence is the binding site of miR-27b in $3^{\prime} U T R s$ of swine RUNX1, the middle sequence is the mature miR-27b, and the lower sequence is the mutated sequence of $3^{\prime}$ UTR. The seed sequence is underlined. (B) Schematic drawing of the putative binding sites or mutations of miR-27b binding-sites in 3' UTR of RUNX1 mRNA. The locations of the potential binding sites or their mutations are presented by blank boxes. (C) The RUNXI luciferase reporter construct was co-transfected with miR-27b mimics (or negative control) or miR-27b inhibitors (or negative control) into PK-15 cells (normalized to the firefly luciferase activity). Data are expressed as relative luciferase activities to control. (D) Western blot analysis of RUNX1 in cells transfected with miR-27b mimics or mimics control. Data represent means \pm S.D. of three independent experiments. $* P<0.05$ in comparison with the control. ${ }^{*} *$ $\mathrm{P}<0.01$ in comparison with the control. 

3'UTR ( 291-311)
5'-UUCCCAGAGGGAAACUGUGAA-3'
ssc-miR-27b 3'-CGUCUUGAAUCGGUGACACUU-5'
3'UTR mutant 5'-UUCCCAGAGGGAAAAUCUCAA-3'
3'UTR(317-337)
5'-CUGAUUUAGCAAUGCUGUGAA-3'
ssc-miR-27b
3'-CGUCUUGAAUCGGUGACACUU-5'
3'UTR mutant
5'-CUGAUUUAGCAAUGAUCUCAA-3'

$\mathrm{C}$
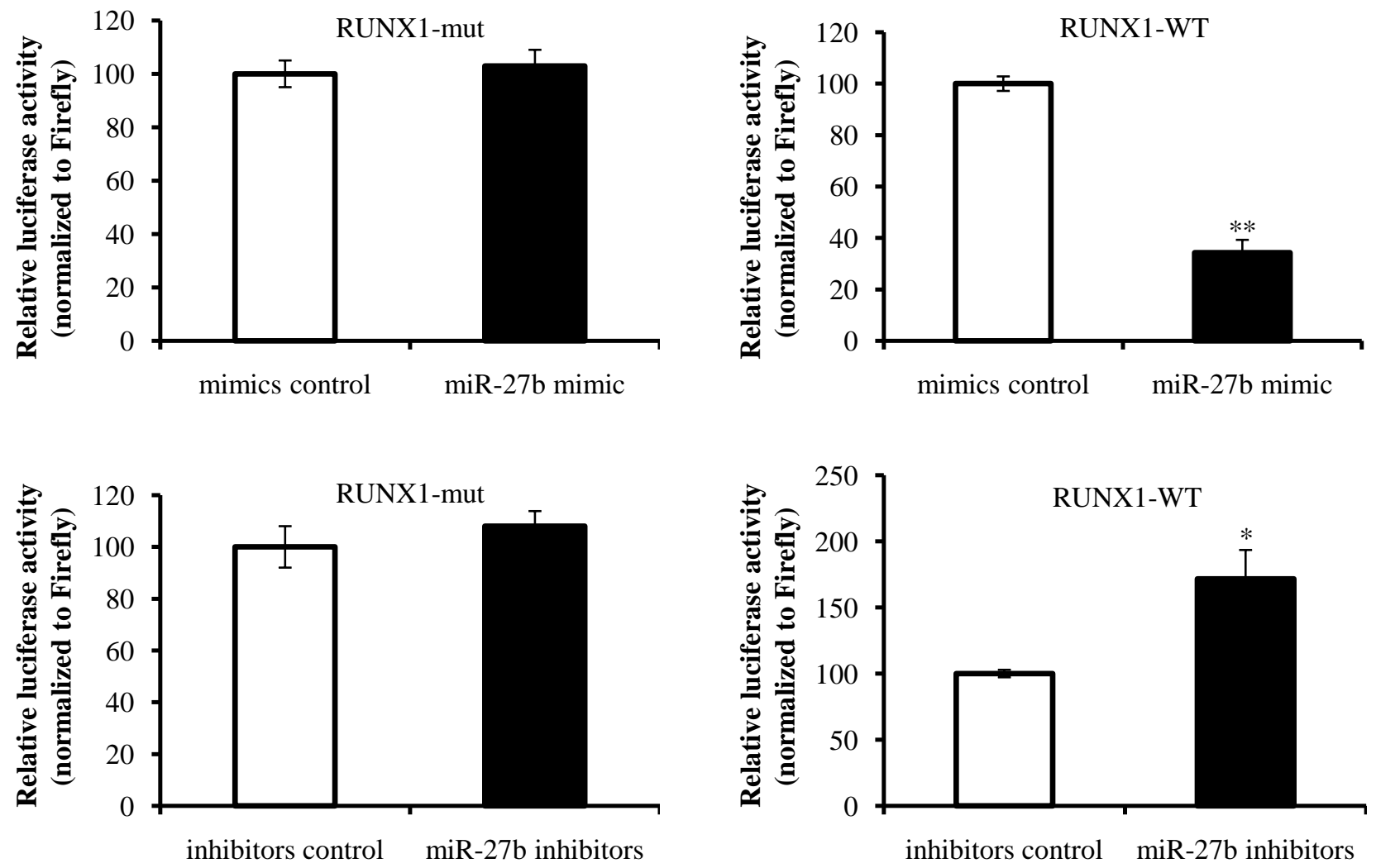

$\mathrm{D}$

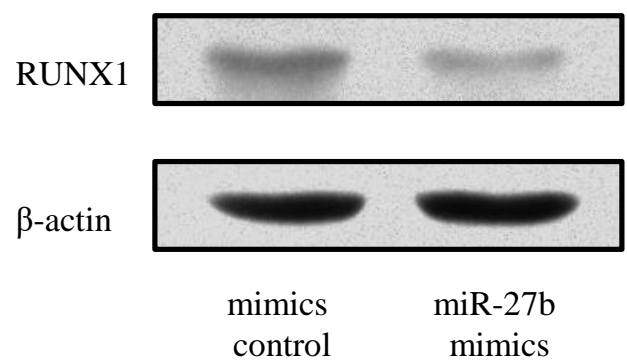


Figure 3 (on next page)

Fig. 3. miR-27b attenuates apoptosis via mitochondrial pathway.

(A) Western blot analysis of Bax in cells transfected with miR-27b mimics or miR-27b inhibitors. (B) Real-time PCR analysis of the expression of Bax in cells transfected with miR27b mimics or miR-27b inhibitors. The Bax mRNA level was reduced at 24 and $48 \mathrm{hpi}$ measured by real-PCR (normalized to $\beta$-actin). Data represent mean $\pm S$.D. of three independent experiments. $* \mathrm{P}<0.05$ in comparison with the control. $* * P<0.01$ in comparison with the control. 
A

PeerJ

\section{Manuscript to be reviewed}

$$
24 \mathrm{hpi}
$$

48 hpi

Bax

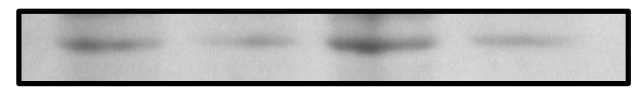

$\beta$-actin

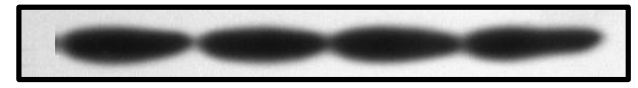

mimics control

miR-27b mimics

1 MOI TGEV
Bax

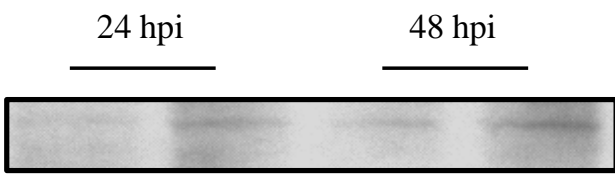

$\beta$-actin

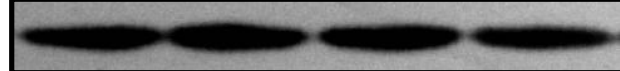

B
24 hpi
48 hpi

24 hpi

48 hpi
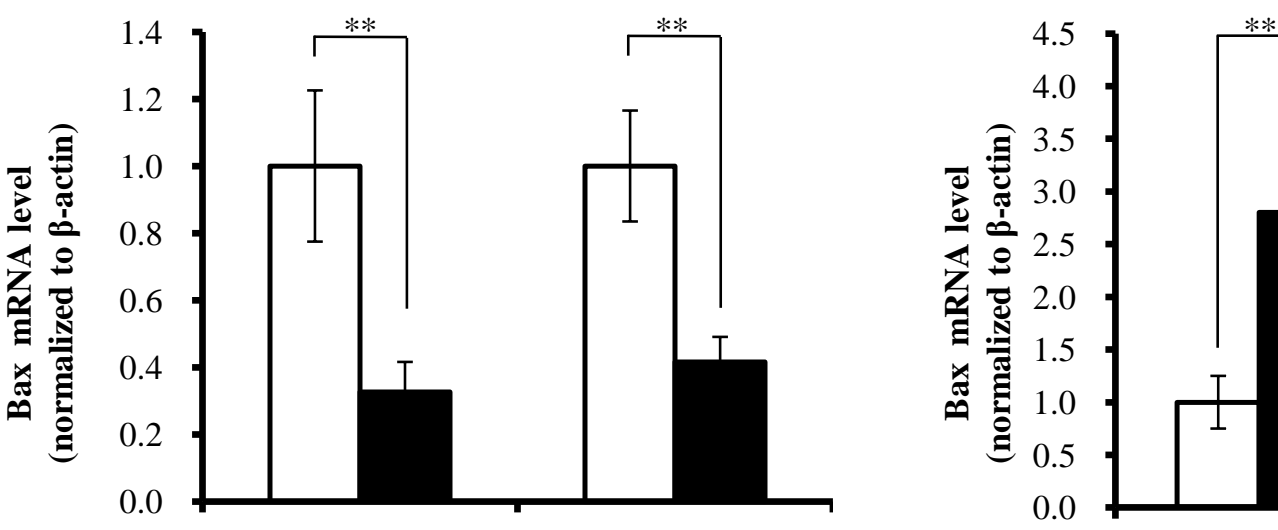

$\begin{array}{lll}- & + & - \\ + & - & + \\ + & + & +\end{array}$

mimics control

miR-27b mimics

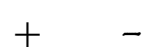

$+\quad-$

inhibitors control

1 MOI TGEV

- $\quad+\quad$ miR-27b inhibitors

$+\quad+$

1 MOI TGEV

$+\quad-$

$-\quad+$

$+\quad+$ 


\section{Figure 4 (on next page)}

Fig. 4. RUNX1 enhances TGEV-induced apoptosis.

(A) Silencing effect of RUNX1 siRNAs on RUNX1 at mRNA level. PK-15 cells were transfected with RUNX1-specific siRNA or irrelevant siRNA and measured by real-time PCR (normalized to $\beta$-actin). (B) The silencing effect of siRUNX1-2 on RUNX1 expression. (C) The effect of siRUNX1-2 on PK-15-cell viability. The cells were incubated after transfecting with $100 \mathrm{nM}$ siRUNX1-2 for $48 \mathrm{~h}$. Cell viability was evaluated by CCK-8 assay. (D) The over-expression of RUNX1 using pCl-neo-RUNX1. PK-15 cells were transfected with pCI-neo- RUNX1 or pCl-neo vector, and the expression level of RUNX1 was assessed by western blot at $48 \mathrm{hpt}$. (E) The effect of RUNX1 on the expression of Bax. (F) The effect of RUNX1 on caspase-9 activity. (G) The effect of RUNXI on caspase-3 activity. Data represent mean \pm S.D. of three independent experiments. $* \mathrm{P}<0.05$ in comparison with the control. $* * \mathrm{P}<0.01$ in comparison with the control. 
Figure 4

PeerJ

A

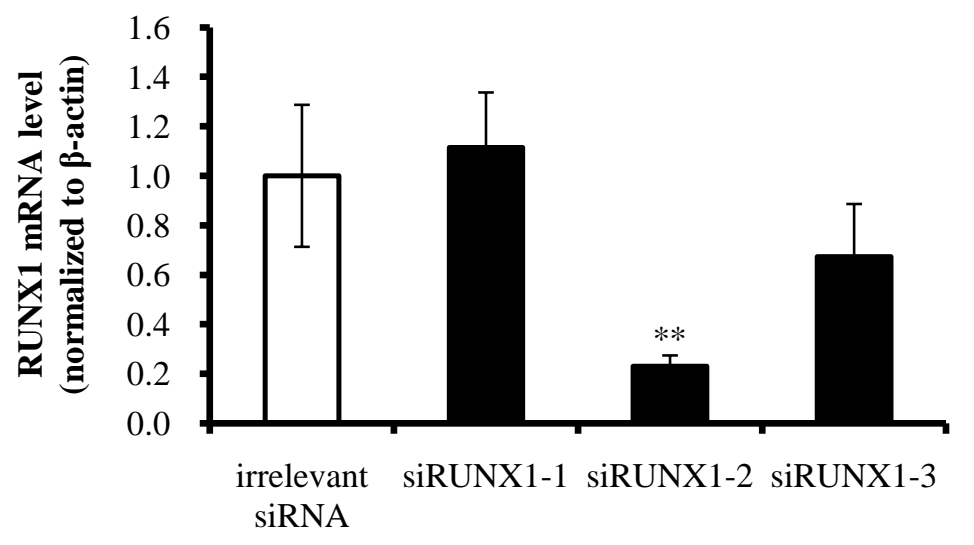

B

Manuscript to be reviewed

RUNX1

$\beta$-actin

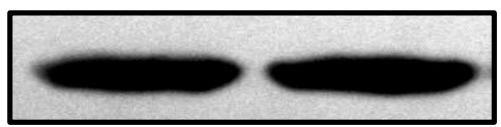

irrelevant siRNA

C

D
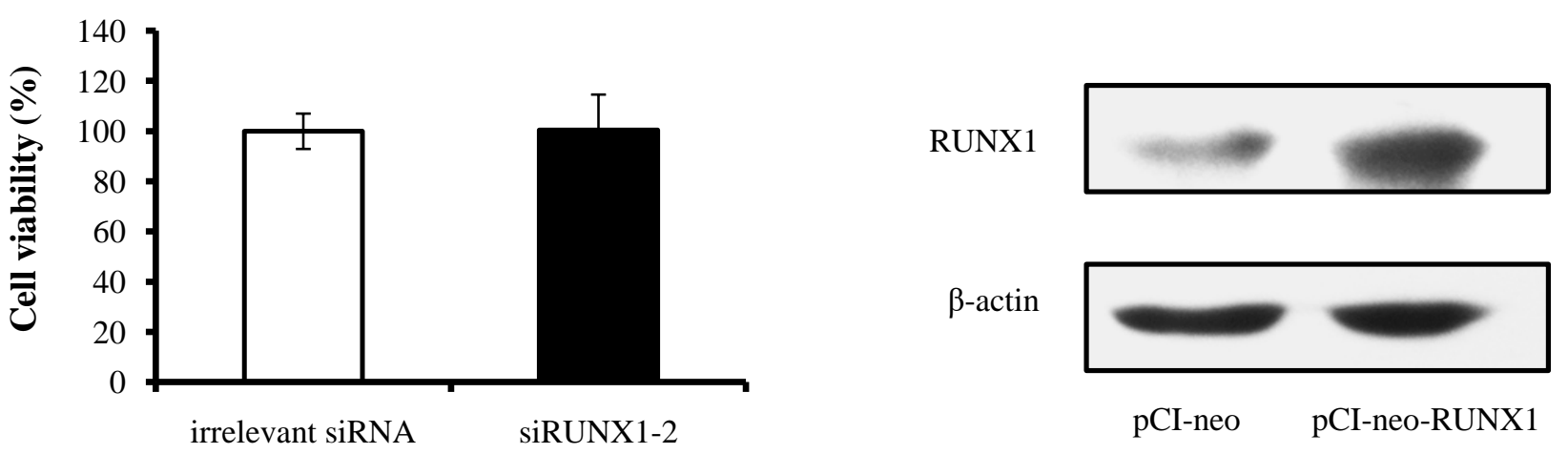

E
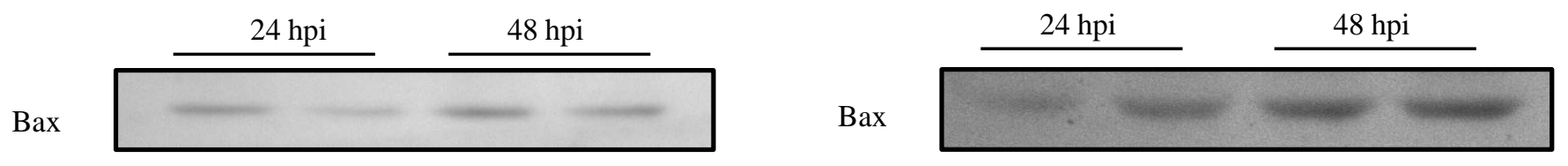

$\beta$-actin

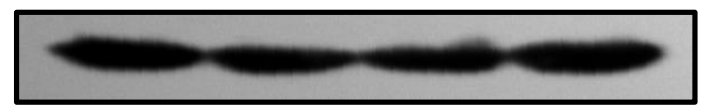

$\beta$-actin

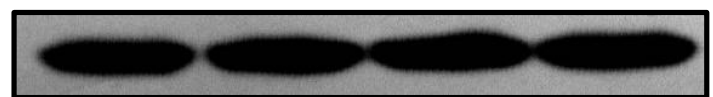

$\begin{array}{rllll}\operatorname{rrelevant} \text { siRNA } & + & - & + & - \\ \text { siRUNX1-2 } & - & + & - & + \\ 1 \text { MOI TGEV } & + & + & + & +\end{array}$

$\begin{array}{rcccc}\text { pCI-neo } & + & - & + & - \\ \text { pCI-neo-RUNX1 } & - & + & - & + \\ 1 \text { MOI TGEV } & + & + & + & +\end{array}$


Figure 4
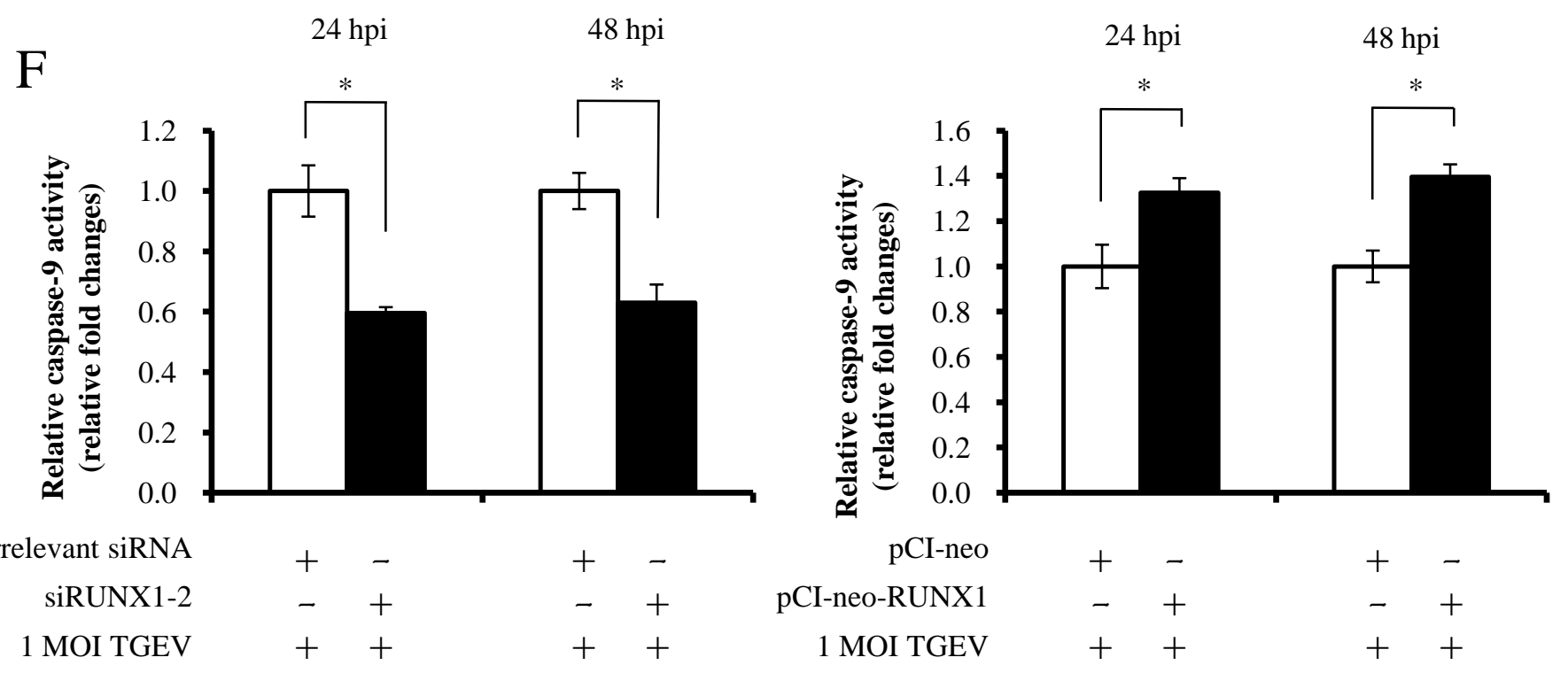

G

irrelevant siRNA

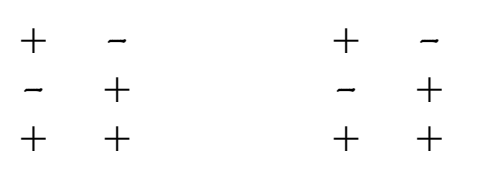

1 MOI TGEV

$+7$

24 hpi

48 hpi

24 hpi

48 hpi

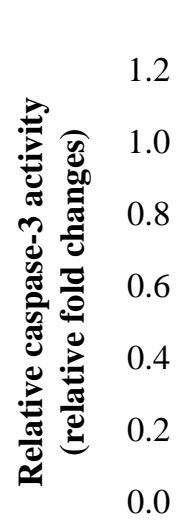

irrelevant siRNA

siRUNX1-2

1 MOI TGEV

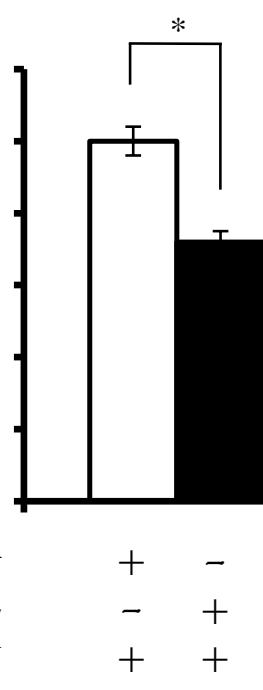

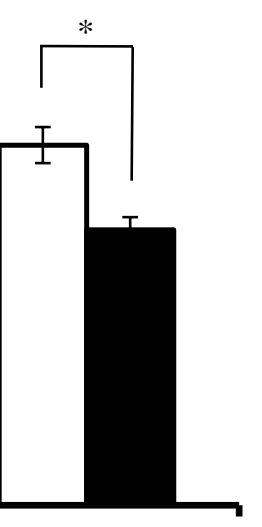

$+\quad-$

$-+$

$++$

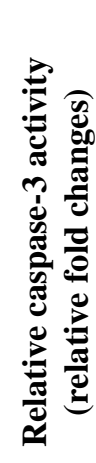

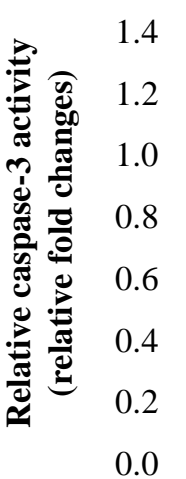

pCI-neo

pCI-neo-RUNX1

1 MOI TGEV

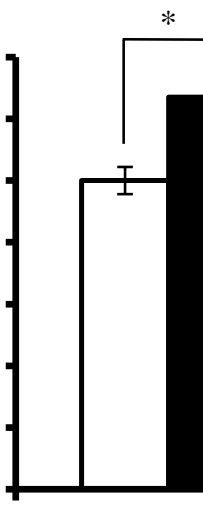

$+\quad-$

$-\quad+$

$+\quad+$

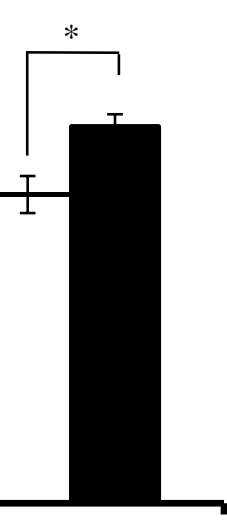

$+\quad-$

$-+$

$+\quad+$ 